\title{
Los principios didácticos constructivistas como prácticas inclusivas en el aula de primaria
}

Karen Palma Rojas

Profesora de apoyo académico y curricular de la carrera Educación General Básica ly ll Ciclos, UNED, Costa Rica; kapalma@uned.ac.cr

\begin{abstract}
Resumen
El sistema educativo costarricense se distingue por atender grupos heterogéneos. Ignorar la pluralidad de los escenarios educativos crea tolerancia a la exclusión porque resta importancia a las necesidades e intereses individuales del estudiantado. La educación inclusiva instaura saberes hacia la diversidad y la expone como una característica propia del ser humano. Promueve prácticas que transforman las metodologías de enseñanza en acciones que se sustentan de lo particular, aumentando en la persona su motivación por aprender y pertenecer a un contexto educativo. Los principios didácticos constructivistas se consideran prácticas inclusivas que articulan las acciones pedagógicas al nivel y ritmo de aprendizaje de los estudiantes. El objetivo del artículo es analizar la metodología de enseñanza inclusiva que se establece a partir del uso constante de principios didácticos constructivistas en un cuarto año de la Escuela José Figueres Ferrer, San José, Costa Rica. Los cinco principios didácticos incluidos fueron: aprendizaje cooperativo, enseñanza explícita, activación de los conocimientos previos, estrategias de metacognición y modelaje cognitivo. Se concluye que los principios didácticos reconocen la diversidad como una oportunidad y no una desventaja; las diferencias individuales no son notorias durante el proceso de enseñanza y aprendizaje porque cada persona aporta desde sus posibilidades. Su uso reveló características de liderazgo, creatividad y autonomía en el estudiantado al asumir aspectos clave en su proceso de construcción del aprendizaje.
\end{abstract}

Palabras clave: Educación básica, educación inclusiva, proceso de enseñanza, método de enseñanza, principios didácticos constructivistas, enseñanza en equipo.

\begin{abstract}
The constructivist teaching principles as inclusive practices in the primary classroom

The Costa Rican educational system is distinguished by attending heterogeneous groups of students. Ignoring the plurality of educational scenarios creates tolerance to exclusion because it detracts from the individual needs and interests of the student body. Inclusive education establishes knowledge towards diversity and exposes it as a characteristic of the human being.

It promotes practices that transform teaching methodologies into actions that are based on the particular, increasing the person's motivation to learn and belong to an educational context. The constructivist didactic principles are considered inclusive practices that articulate the pedagogical actions to the level and rate of learning of the students. The objective of this article is to analyze the methodology of inclusive teaching that is established from the constant use of didactic constructivist
\end{abstract}


principles in a fourth year of José Figueres Ferrer School, San José, Costa Rica. The five didactic principles included: cooperative learning, explicit teaching, activation of previous knowledge, strategies of metacognition and cognitive modeling. It is concluded that the didactic principles recognize diversity as an opportunity and not a disadvantage; the individual differences are not obvious during the process of teaching and learning because each person contributes from their possibilities. Its use revealed characteristics of leadership, creativity and autonomy in the student body by assuming key aspects in its process of learning construction.

Key words: Basic education, inclusive education, teaching process, teaching method, teaching principles constructivist, teaching as a team.

\section{INTRODUCCIÓN}

El sistema educativo costarricense se distingue por atender grupos heterogéneos de estudiantes en edad, religión, procedencia, emociones, costumbres y estilos de aprendizaje. La pluralidad es una cualidad de los escenarios educativos y omitirla ocasiona que la persona estudiante se sienta excluida, pues no se consideran sus necesidades e intereses, únicamente se le pide cumplir patrones estandarizados de rendimiento, aprendizaje y madurez.

Ofrecer una educación de calidad solicita ver la diversidad como riqueza y no como limitación:

(...) dos son las características fundamentales que debe tener nuestro modelo de escuela del presente para un futuro inmediato: calidad y equidad... La calidad de la educación sólo puede alcanzarse si llega a todos y es de calidad para todos, sin excepciones ni discriminación, así la equidad en educación se convierte en un criterio de calidad. (Muntaner, 2014, p.64)

Implementar el indicador de calidad y equidad mencionado por Muntaner, requiere de un profesional convencido del respeto de los derechos humanos y la naturaleza de la educación inclusiva, la que busca rescatar las diferencias de cada persona y las sitúa como aportes que enriquecen el proceso de enseñanza y aprendizaje.

La implementación de una educación inclusiva articula dos requerimientos para el sistema educativo: ofrecer un currículo común que acoja una amplia población en respuesta a los intereses y necesidades económicas de un país y reconocer características individuales en cada persona para facilitar y favorecer así su aprendizaje (Escudero y Martínez, 2011).

La educación inclusiva busca instaurar consciencia y respeto a la diversidad, "lo que se traduce en la no categorización de los alumnos bajo ningún criterio... acabando definitivamente con...la falacia de la homogeneidad y uniformidad de los grupos" (Muntaner, 2014, p.66). En respuesta a lo anterior, se promueven prácticas inclusivas que (entre muchas de sus funciones) transforman las metodologías de enseñanza en acciones que involucran a todo el estudiantado; estimulando así: la construcción del conocimiento, la motivación, el sentido de pertenencia y la satisfacción hacia el aprendizaje logrado.

Los principios didácticos constructivistas pueden pensarse como prácticas inclusivas porque incluyen los criterios de calidad y equidad antes descritos; permiten al docente acoplar las acciones de enseñanza al nivel y ritmo de aprendizaje de sus estudiantes "el objetivo es claro, el papel del maestro es guiar, orientar, mediar para que el estudiante penetre en el mundo de la cultura sistematizada, pero a partir de lo propio" (Alfaro, Arauz, Avendaño y Rojas, 2000, citados por Abarca, 2007, p. 115). 
El artículo analiza la experiencia de implementar prácticas inclusivas en la escuela primaria pública donde se incorporan principios didácticos constructivistas en las metodologías de enseñanza impartidas a un cuarto año de la Escuela José Figueres Ferrer ubicada en San José, Costa Rica.

\section{JUSTIFICACIÓN}

La educación costarricense se ha distinguido a través de los años por ser pública, gratuita y obligatoria, pero esto no garantiza la calidad de los procesos de enseñanza que ofrece. La mayoría de los centros educativos de primaria y secundaria del país se caracterizan por desarrollar sus lecciones desde la modalidad "magistral", donde el docente (encargado de dirigir todo el proceso) entrega información que el estudiante reproduce en un ejercicio, práctica o prueba escrita. Reafirmando lo anterior, $72 \%$ de los docentes indican que es poco el tiempo que poseen para impartir sus lecciones, por lo cual destinan la mayor cantidad de éste, en explicar el tema que se estudia, resolver ejercicios en la pizarra, revisar trabajos de clase o dar instrucciones a sus estudiantes (Estado de la Educación, 2011).

Las prácticas de aula mencionadas se denominan exclusivas porque reproducen un mismo patrón de enseñanza para todos y como resultado de su uso generan vacíos importantes en los aprendientes, ocasionando problemas para entender un tema, recordarlo y aplicarlo en un futuro; la ineficiencia de la acción pedagógica causa rupturas en el aprendiente difíciles de sanar por largo tiempo.

Convertir la educación en un instrumento útil y de transformación social, solicita admitir otras metodologías de enseñanza que proporcionen momentos activos y significativos para aprender. El diseño universal del aprendizaje (DUA) presenta un concepto novedoso y avanzado de educación, al señalar que su meta final es formar "aprendices expertos-individuos que saben cómo aprender, qué quieren aprender, y quienes, a su manera, están preparados para una vida de aprendizaje" (CAST, 2008, p.3).

El DUA presenta tres aspectos fundamentales que reorientan el quehacer educativo: 1. centralizar los procesos de enseñanza en el aprendiente; 2. trabajar con la persona desde una perspectiva inclusiva, sin importar su procedencia o condición; 3. visionar la filosofía del "aprender a aprender" como una herramienta que le da autonomía a la persona porque logra a futuro resolver cualquier problemática.

En respuesta de lo anterior, los principios didácticos constructivistas permiten el desarrollo de la educación que el DUA solicita al centrar la enseñanza en el estudiante, porque promueven "estrategias de enseñanza flexibles e innovadoras que permiten una educación personalizada, reflejada en el reconocimiento de estilos de aprendizaje y capacidades entre los estudiantes...ofertan diferentes alternativas de acceso al conocimiento" (Ministerio de Educación Nacional [M.E.N], 2009, p.10).

\section{SUSTENTACIÓN TEÓRICA}

\section{La educación inclusiva}

Se concibe la educación inclusiva como un conjunto de acciones que abren los contextos a la plena participación de todos en igualdad de condiciones, el estudiantado se da cuenta de que sus necesidades y objetivos personales son considerados durante los procesos de enseñanza y aprendizaje. Busca el respeto de los derechos humanos y crea sentido de pertenencia en los miembros de la comunidad educativa (estudiantes, personal docente y administrativo; padres de familia, miembros de la comunidad, entre otros) con la institución. 
La educación inclusiva transforma el currículo tradicional en flexible al promover respeto por lo particular en los procesos cognitivos de cada estudiante. Las metodologías de enseñanza reconocen que todo lo que ocurre en el salón de clases posee gran significado y utilidad para la vida; no sólo se procura la construcción del conocimiento, sino que también, se busca el desarrollo de habilidades sociales que permitan la sana convivencia en cualquier contexto social: valores, hábitos de convivencia, estímulo de la ética, entre otros.

Para Calero (2008, p.146) este tipo de pedagogía "es paidocentrista, lo que significa que el estudiante debe ser el protagonista de la acción educativa". El docente inclusivo desarrolla metodologías de enseñanza que rompen barreras de aprendizaje y participación creadas desde la escuela tradicional. Para el autor, este tipo de educación redefine el actuar de los centros de educativos al implementar criterios que promueven:

a. Estrategias de motivación: se busca a través de ellas convertir la motivación extrínseca en intrínseca, al considerar los intereses y necesidades que motivan el aprendizaje en cada estudiante.

b. Metodologías activas: el docente investiga y respeta la manera en que todo estudiante capta la información, las actividades de clase benefician cualquier estilo de aprendizaje.

c. La formación en habilidades sociales fomenta el trabajo en equipo: se promueve la armonía y el sano convivir dentro de la diversidad, como estados afectivos que le permiten al estudiantado sentirse motivado por aprender.

d. La filosofía de aprender a aprender: permite al estudiante organizar, analizar, comparar, (entre otras habilidades cognitivas) la información brindada en la clase.

e. El cambio en la organización escolar: para fortalecer el sentido de pertenencia de la comunidad educativa, el personal docente planifica las actividades del centro educativo considerando las características de la población estudiantil. Las actividades pueden ser actos cívicos, celebración de efemérides, fiestas escolares, paseos, entre otros.

\section{Los principios didácticos constructivistas}

La didáctica se define como el área de la pedagogía que brinda una variedad de métodos y técnicas de enseñanza al docente, para que cumpla con los objetivos de instrucción propuestos (López, 2008). La teoría constructivista nombra principios didácticos a estas técnicas y métodos de enseñanza, porque permiten aplicar los fundamentos y máximas del constructivismo en el contexto educativo (Díaz y Hernández, 2002).

Los principios didácticos tienen "el objetivo...de darle [al estudiante] oportunidades de pensar, de aprender y de obrar de manera independiente" (Calero, 2008, p.53). Por esto, cuando el estudiante se apropia de ellos logra vivir el autoconocimiento de sus procesos de cognición, obtiene autonomía al aprender desde la "toma de decisiones consciente e intencional... elige y recupera los conocimientos que necesita para hacer su trabajo" (Arias, Coronado, Rolla, Romero y Rivadeneira, 2011, p.30).

A continuación se describen cinco de los muchos principios didácticos de la teoría constructivista: aprendizaje cooperativo, enseñanza explícita, activación de los conocimientos previos, estrategias de metacognición y modelaje cognitivo. Se eligieron estos por abrir espacios de participación, diálogo y análisis entre el estudiantado y la docente de grupo. 


\section{Aprendizaje cooperativo}

El aprendizaje cooperativo hace referencia a la organización de los estudiantes en equipos de trabajo, impregna otro sentido y dinámica al proceso de construcción del conocimiento, promueve que todas las personas actúen para maximizar su aprendizaje y el de sus compañeros. Los equipos pueden estar conformados por sujetos que pertenecen a un mismo ambiente educativo: aula, profesorado, escuela, colegio, etc. (M.E.N., 2009).

El aprendizaje cooperativo promueve la construcción del conocimiento bajo redes de apoyo heterogéneas, beneficia la socialización entre pares y establece relaciones de colaboración, porque desarrolla "conductas prosociales(...) que mantiene un clima de respeto y valoración de las diferencias" (Calero, 2008, p.169).

Hernández (2004) llama a este principio didáctico "mediación entre pares", indica que enriquece de muchas formas a la persona involucrada, porque durante el intercambio con sus pares, se adquiere una comprensión profunda de los demás. Menciona que los miembros de un grupo comparten un mismo nivel de confianza, vocabulario y experiencias previas, creando estilos únicos para explicar, comprender la teoría y resolver las actividades propuestas.

\section{Enseñanza explícita}

Consiste en enseñar mediante descripciones absolutamente claras lo que el profesional quiere lograr (objetivos), lo que se va a hacer (actividad) y el porqué de eso (raciocinio) (Rubio y Álvarez, 2010), permite dar sentido a lo que se estudia, preparando a la persona para las actividades que vivirá durante la clase. El vocablo "explícito" significa expresar clara y determinadamente lo que desea lograr, admite que el docente entregue (de cierta forma) el control de su clase, porque antes de iniciar una actividad o experiencia, el estudiante conoce todo lo referente al trabajo que se ha planificado. La enseñanza explícita, para Arias, et al. (2009):

(...) resulta muy efectiva, debido a la conciencia o el grado de alerta del estudiantado acerca de lo que está aprendiendo, las acciones que emprenderá para construir el aprendizaje, y sobre todo, la utilidad específica [que tendrá este aprendizaje] en diversos contextos. (p.56)

Desde lo anterior, se genera una educación paidocentrista (Calero, 2008) porque durante la explicación que el docente ofrece sobre las tres preguntas claves (por qué, para qué y cómo), se le da un papel principal al estudiante en su proceso de enseñanza y aprendizaje, el mediador deja atrás la idea de que el estudiante es únicamente un receptor y busca despertar en ellos curiosidad hacia el tema de estudio o la actividad por desarrollar.

La enseñanza explícita puede emplearse en dos momentos:

a. Preparatorio del día: antes de iniciar las actividades del día, el docente presenta una clara y amplia descripción de los temas por desarrollar, el objetivo y alcance de las actividades, así como sus expectativas como pedagogo, explicando el cuándo, cómo y por qué de cada actividad.

b. Preparatorio para cada asignatura: es cuando se profundiza en las actividades por desarrollar en cada periodo lectivo. Permite que el estudiante comprenda con detalle el qué, por qué y cómo de cada tema y actividad.

\section{Activación de conocimientos previos}

Este principio didáctico elimina la vieja concepción que el docente es el único experto sobre el tema que se estudia, reconoce que todos los integrantes de la clase conocen el tema, pero en diferentes niveles. La 
activación de conocimientos previos es el primer paso para la construcción de un nuevo conocimiento, porque "el aprendizaje llega a ser un proceso constructivo interno (...) es decir, que el punto de partida será siempre los conocimientos previos" (López, 2008, 10).

Su aplicación es muy sencilla, radica en llevar a la persona a vivir la experiencia de cuestionarse qué sabe sobre un tema; para ello el docente indaga cuál es la representación o ideas espontáneas que posee el estudiantado al respecto. Para su logro se utilizan actividades como: periodos de conversación, preguntas generadoras, entrevistas, lluvias de ideas, mapas conceptuales, diálogos, entre otros.

\section{Modelaje cognitivo}

El modelaje cognitivo consiste en que el docente o el estudiante exterioricen cómo solucionan una actividad o aplican una estrategia; quien modela habla para sí mismo, explica con detalle sus pensamientos y los pasos que sigue para cumplir una tarea. Para Good y Braphy (1996, citado por M.E.N., 2009) "El modelamiento es una forma de aprendizaje en la que los individuos imitan la conducta de otros después de observarlos...el aprendizaje por medio de esta estrategia es más exitoso cuando el profesor forma relaciones personales con sus estudiantes" (p. 403).

Este principio didáctico se puede aplicar en tres niveles, respetar el orden en que se presenta cada nivel esclarece el uso e incorporación de nuevos aprendizajes. A continuación se describe cada nivel:

a. Primer nivel "modelaje perceptual", la persona docente hace modelaje de la tarea frente al resto de la clase y ellos observan en silencio. Mientras habla en voz alta consigo mismo, también reflexiona sobre los pensamientos que llegan a su mente, transmitiendo estrategias de reflexión y la toma de decisiones. Puchol (2010) llama a esta etapa modelado pasivo, porque el estudiante únicamente observa la conducta del modelo, sin reproducirla durante la sesión de entrenamiento.

b. Segundo nivel "modelaje participativo", el docente modela frente al estudiantado que participa activamente dando ideas de cómo pueden ejecutar los diferentes momentos de la tarea. Aquí las intervenciones del estudiante le indicarán al docente la comprensión que posee de la estrategia o tarea por ejecutar, lo cual garantiza que efectivamente el estudiantado este adquiriendo procesos hacia la autonomía de su aprendizaje, justificando el tiempo a invertir en la segunda etapa. Esta fase se podrá repetir cuantas veces se considere necesario para pasar al tercer nivel.

c. Tercer nivel "modelaje recíproco", siguiendo el formato del anterior nivel, la ejecución y construcción de la estrategia la realizan con igual responsabilidad estudiantes y docente. Uno o dos estudiantes inician el modelaje y los espectadores comparten ideas para la elaboración del trabajo; poco a poco el docente retira su ayuda, para que por sí solos los estudiantes logren deducir cómo se ejecuta la estrategia. Puchol (2010) le llama a la etapa modelaje activo, porque el sujeto observa y a continuación reproduce solo la tarea antes modelada.

\section{Metacognición}

Principio didáctico referido a la conciencia que desarrolla la persona sobre sus procesos de aprendizaje, Arias et al. (2011) la define de la siguiente forma:

El conocimiento de los propios procesos cognitivos...se refiere al estar atento y consciente de cómo se construye ese conocimiento...es la conciencia que tiene el sujeto sobre lo que aprende, para qué lo aprende y las actitudes respecto a ese aprendizaje. La metacognición se refiere al control consciente de los procesos cognitivos como la atención, la memoria y la comprensión (p.20). 
Utilizar la metacognición como estrategia didáctica, le brinda al estudiante herramientas para tomar el control de su aprendizaje (aprender a aprender), le otorga autonomía de aprendizaje-enseñanza, por lo tanto en la revisión, organización y evaluación de las actividades logra por sí solo detectar cómo estuvo su desempeño, identificando los errores que cometió y reflexionando sobre el porqué de ellos. Al mismo tiempo, el estudiantado adquiere conciencia de los nuevos conocimientos adquiridos (M.E.N., 2009).

Bajo este principio didáctico, el aprendizaje es estratégico porque "los aprendices [logran ser] conscientes de sus esfuerzos al utilizar habilidades y estrategias peculiares descubiertas por ellos mismos" (Jones, 1997, citado por Hernández, 2004, p. 7). En el futuro utilizarán esas mismas acciones para continuar controlando su aprendizaje.

\section{PROPUESTA TRANSFORMADORA DE LAS METODOLOGÍAS DE ENSEÑANZA}

\section{Contexto escolar}

La experiencia de implementar cinco principios didácticos de la teoría constructivista para transformar las metodologías de enseñanza en inclusivas, se realiza en un cuarto año de la escuela José Figueres Ferrer del circuito 03, de la Dirección Regional de Educación, San José Norte.

En el año 2014, la sección 4-1, estaba conformada por veinticinco estudiantes, trece mujeres y doce varones, en edades de 9 a 11 años. Este grupo estudió junto desde preescolar. Durante el primer ciclo de la escuela primaria fueron atendidos por dos profesoras que aplicaron el aprendizaje cooperativo como metodología de trabajo. Como resultado, durante la aplicación de esta experiencia, el estudiantado ya conocía el uso y roles de trabajo requeridos.

La incorporación de principios didácticos constructivistas como apoyo pedagógico y prácticas inclusivas se realizó en las asignaturas "básicas" que impartía la docente guía (Español, Matemática, Ciencias y Estudios Sociales), no así en las asignaturas "complementarias" (Educación Física, Computación, Religión e Inglés) por estar a cargo de otras profesionales. La decisión pedagógica de enriquecer las metodologías de enseñanza nació durante la planificación anual (a inicio de curso lectivo), donde la docente a cargo (autora del artículo) toma decisiones relevantes con el objetivo de ofrecer un ambiente inclusivo, atender los intereses del estudiantado y subsanar situaciones (familiares o del sistema educativo) que enfrentan regularmente los estudiantes de cuarto año, como se detallan a continuación:

a. Características propias a su edad: Los estudiantes que cursan el cuarto grado están en un promedio de edad de 9 a 11 años, esto indican que la mayoría presenta características sociales propias de la preadolescencia. Ahora su imagen corporal tiene mayor peso, gustan de compañeros del género opuesto, cuestionan o retan constantemente la autoridad, buscan privacidad para sus situaciones y pensamientos, forman grupos cerrados de amigos donde prefieren relacionarse únicamente con personas de su mismo género y muestran baja tolerancia ante la diversidad (lo que puede agravar situaciones de burla, rechazo o maltrato entre ellos).

b. Nivel de exigencia académico: el cuarto grado se ubica en el segundo ciclo de la Educación General Básica, lo cual aumenta la exigencia académica en las asignaturas de su currículo. El sistema educativo da por hecho que el niño o niña alcanza una madurez cognitiva que les permite comprender conceptos abstractos y lejanos de su realidad inmediata; por lo tanto el docente eleva el nivel de exigencia de las actividades de clase y de las pruebas escritas, por considerar que el estudiantado de cuarto año domina habilidades cognitivas como la síntesis, el análisis, la evaluación, la 
capacidad para memorizar un alto volumen de datos y el manejo fluido de las cuatro operaciones matemáticas básicas.

c. Dinámica familiar: un factor que afecta a muchos estudiantes es que presentan cambios físicos propios de la adolescencia, entonces llegan a ser considerados como pequeños adultos, esto modifica en muchas ocasiones los nexos y comunicación con sus padres o adultos cuidadores. Se cree que muchos ya son capaces de tomar decisiones asertivas y no requieren de vigilancia o apoyo para el estudio; entonces dejan en el niño o niña la responsabilidad casi total de su estudio y baja el apoyo del adulto para prepararse para las pruebas escritas, en la solución de tareas y de trabajos extraclase. Es importante considerar que algunos estudiantes deben asumir tareas del hogar como preparar parte de su comida, cuidar hermanos menores y realizar tareas de limpieza.

\section{Experiencia educativa}

Los cinco principios didácticos fueron incorporados desde el primer día de clases, con el objetivo de beneficiar a los estudiantes en el manejo de los mismos y estimular así las habilidades para el aprendizaje como: el aprender a aprender, la autonomía, la metacognición. Emplear los principios diariamente estableció "rutas didácticas" que permitieron un desarrollo novedoso de la clase. La figura 1 muestra las tres rutas didácticas que surgieron con el uso de estas prácticas inclusivas.

\section{Actividades diarias del círculo}

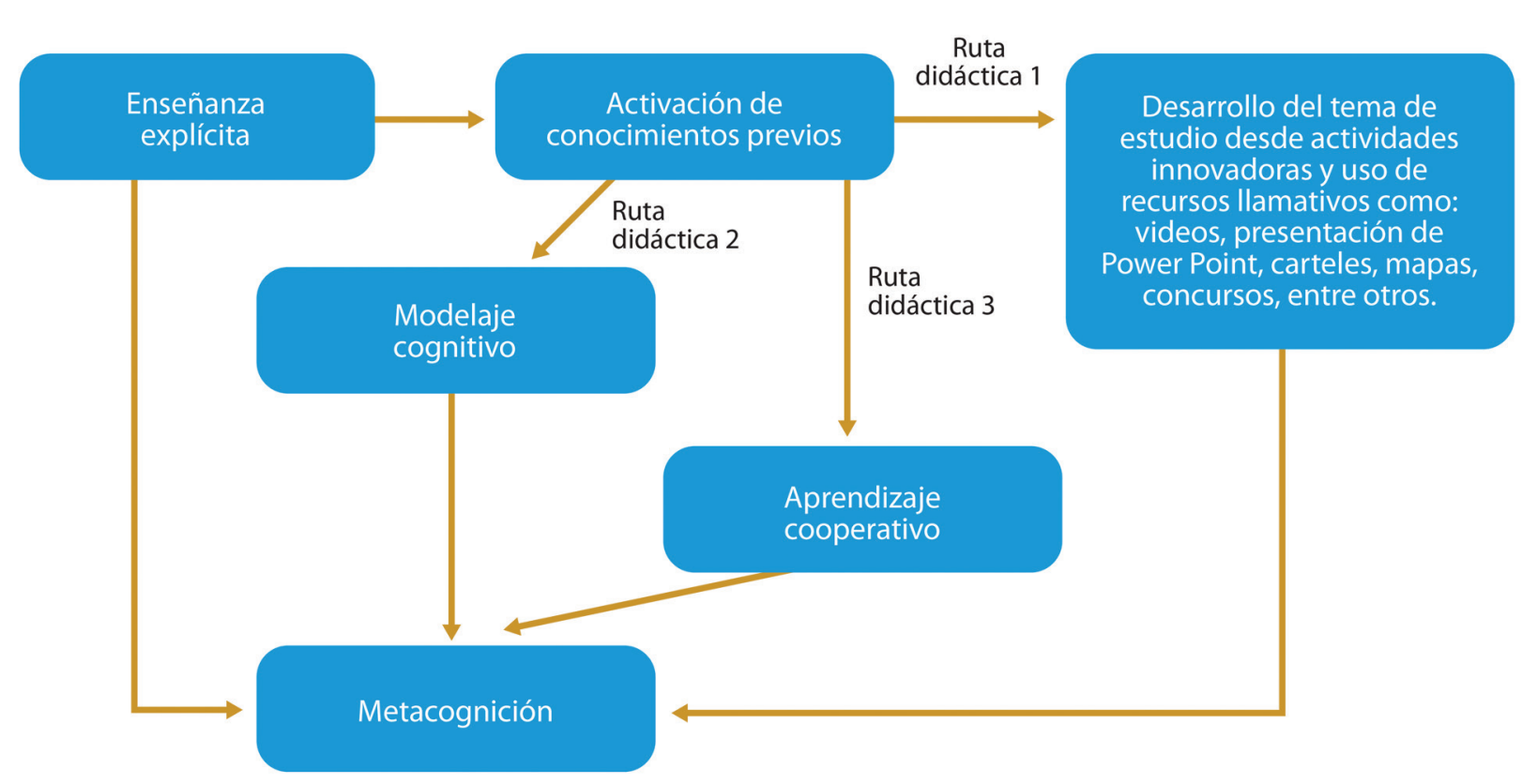

Figura 1. Rutas didácticas que surgen desde la incorporación de los principios didácticos constructivistas en la metodología de enseñanza. Fuente: elaboración propia.

El día lectivo iniciaba con el espacio llamado "círculo", su principal objetivo era propiciar momentos de comunicación entre el estudiantado y la docente sobre situaciones de aula, noticias nacionales, cumpleaños o cualquier otro tema que fuera del interés de los niños y las niñas. Para la actividad, se utilizó la estrategia de sentar a todos los miembros de la clase (estudiantes y docente) en el piso del aula, para 
crear cercanía y romper con la rutina del trabajar sólo en los pupitres. Además, la proximidad le permitía a la docente comprobar que todos estuvieran prestando atención y comprendieran la clase en su desarrollo. Luego, se iniciaba el uso de los principios didácticos. A continuación se describe el proceso.

\section{Enseñanza explícita}

La enseñanza explícita se aplicaba en dos diferentes momentos, primero durante el "preparatorio del día" y luego en el "preparatorio para cada asignatura". Al final del día lectivo se había empleado de tres a cuatro veces. A continuación se detallará su uso:

a. Preparatorio del día: el círculo de la mañana concluía con la aplicación del principio de enseñanza explícita, los estudiantes conocían este momento como "agenda del día" porque el orden del día lectivo se ilustraba a través de una serie de imágenes que se colocaban al lado de la pizarra principal del aula, cada una representaba el orden de las asignatura (obligatoria o especial) del día. Cuando la docente situaba cada imagen, describía el tema, su importancia, explicaba los objetivos que como profesional establecía para la clase, los aprendizajes que deseaba lograr en los estudiantes, las actividades y los materiales. El momento se aprovechaba para recordar la entrega de alguna tarea, trabajos extraclase, tiempos de recreo y se recapitulaba lo último visto en cada asignatura.

b. Preparatorio para cada asignatura: se utilizó al inicio de cada asignatura y se pedía a los estudiantes estar sentados en el piso para iniciar con el uso de este principio didáctico, lo cual garantizaba la atención de todos y rompía con la rutina de trabajar solamente en los pupitres. De seguido, la docente exponía con detalle las actividades, objetivos e intereses propios para el desarrollo de la asignatura.

\section{Aprendizaje cooperativo}

Como el trabajo en equipos era algo conocido por los estudiantes, las actividades de clase se planificaban casi siempre para cumplir con este principio didáctico. La agrupación de los equipos se realizaba desde tres criterios: 1. avance cognitivo, 2. habilidades y destrezas, 3. relaciones frecuentes. El último criterio se pensó para fomentar la unión de grupo al reunir estudiantes que se relacionaban poco entre sí, que habían tenido alguna diferencia o por ser de género contrario. El estudiantado conocía que su equipo de trabajo variaba según la asignatura y el tipo de trabajo por resolver.

El criterio de agrupación era determinado por la actividad planificada, por ejemplo, en Matemática cuando se trabajaba la resolución de problemas o en Español la comprensión de una lectura, casi siempre se agrupaban los estudiantes por sus avances cognitivos o habilidades y destrezas. Lo anterior con el objetivo de generar redes heterogéneas de apoyo. Para Ciencias o Estudios Sociales se trabajaba por proyectos cortos, entonces se agrupaban los estudiantes mezclándolos bajo el criterio de relaciones frecuentes, porque la actividad propiciaba momentos de negociación y conversación que exigía conocerse y relacionarse entre sí.

Antes de iniciar el trabajo de los equipos, la docente tomaba un tiempo para dar indicaciones relevantes en el cumplimiento del trabajo: tiempo, materiales, lugares del centro educativo que podían utiliza para reunirse y roles de cada integrantes. Para algunas actividades se presentaba una rúbrica para evaluar el trabajo de cada equipo, la cual calificaba aspectos claves para la sana convivencia tales como respeto de los integrantes, cooperación, aportes al logro del trabajo grupal, cumplimiento del tiempo, exposición, entre otros criterios. Cada estudiante copiaba la rúbrica en su cuaderno y la profesora luego de escuchar la exposición de cada grupo y de evaluar el trabajo realizado, colocaba una calificación cualitativa para cada indicador: excelente, muy bueno, bueno y por mejorar. 
Los roles de trabajo que se distribuían entre los integrantes de un equipo eran: coordinador, secretario y colaboradores, estos eran asignados por la docente y en varias ocasiones los miembros proponían los roles.

a. Coordinador: persona encargada de distribuir las tareas de trabajo y vigilar que todos cumplieran con las mismas.

b. Secretario: integrante que tenía la tarea de hacer las consultas que surgían del proceso de construcción de los trabajos. Además era la persona encargada de escribir el trabajo del equipo para luego presentarlo a la docente.

c. Colaboradores: otros integrantes del equipo, cada uno tenía una tarea por cumplir para completar el trabajo asignado al equipo. El estudiantado reconocía que sin los colaboradores no se podía completar el trabajo final y que el aporte de cada uno era muy importante.

El trabajo en equipos concluía (la mayoría de ocasiones) con una exposición. Todos los miembros debían presentar algún aspecto del trabajo elaborado, para lo cual los niños y niñas realizaban carteles o esquemas. Luego cada integrante explicaba a los demás cuáles fueron sus aportes para el logro de la tarea y qué aprendió. Al finalizar, la profesora completaba la rúbrica para cada miembro del grupo.

\section{Activación de conocimientos previos}

La activación de los conocimientos previos insta al estudiante a construir nuevos aprendizajes desde el uso de otros principios didácticos como el aprendizaje cooperativo, el modelaje cognitivo y la metacognición. En el momento de esclarecer viejos saberes se utilizaron actividades y recursos activos como videos cortos, escribir una palabra o una frase en la pizarra y luego analizarla, leer un fragmento de una noticia o solicitarle a un estudiante contar qué conoce de un tema para luego despertar una lluvia de ideas en el resto del grupo. El principio didáctico se utilizaba varias veces dentro de una misma clase, las interrogantes que hacía el estudiantado o un equipo de trabajo, daba pistas a la docente sobre qué tema debía activar nuevamente durante la clase.

\section{Modelaje cognitivo}

El modelaje se utilizaba para mostrar a los estudiantes estrategias que permitieran descubrir rutas propias en la construcción de nuevos conocimientos, formarse sobre otro tema y fortalecer los aprendizajes ya alcanzados. Varios ejemplos de estrategias modeladas ante los estudiantes son: ordenar las ideas para escribir un texto, sintetizar una lectura, hallar la operación matemática para solucionar un problema, dividir con una cifra y multiplicar por dígitos del 10, entre otras muchas.

Durante la aplicación del modelaje cognitivo, se respetó el orden de los tres niveles, el paso a uno superior lo indicaba la comprensión y apropiación mostrada por la mayoría de estudiantes para cada estrategia. Cuando un grupo de niños y niñas revelaba haber asimilado una estrategia, la docente aprovechaba para solicitarles a algunos de ellos modelar desde el segundo nivel (participativo) y luego pedía a otros estudiantes modelar desde un tercer nivel (recíproco). En algunos momentos se solicitó también a un equipo modelar desde el nivel participativo, ellos explicaban cómo lograron desarrollar y aplicar la estrategia en la actividad de clase.

\section{Metacognición}

La metacognición se empleó durante tres momentos clave de la clase:

a. La enseñanza explícita: cuando la docente explicaba el porqué de la actividad o tema por desarroIlar, hacia comentarios intencionados para despertar la toma de conciencia hacia procesos mentales 
que cada estudiante utilizaba al resolver un ejercicio, actividad o comprender un nuevo tema. Adicionalmente se advertía qué momentos de la clase eran clave para detenerse y reflexionar sobre rutas personales al adquirir el aprendizaje.

b. El modelaje cognitivo: durante el nivel recíproco, la docente indagaba en el modelador cómo logró comprender la estrategia. La pregunta generadora era ¿qué pasos seguiste en tu mente para comprender que esto se solucionaba o se realizaba de ese modo? con la interrogante muchos niños y niñas autoreflexionaban sobre qué estrategias utilizaban para aprender, además el resto del grupo podía verse tentado en contestarse a sí mismos y concientizar así sus procesos metacognitivos.

c. El aprendizaje cooperativo: para el momento de la exposición de cada equipo, la docente intervenía preguntándole a cada integrante ¿cómo logró comprender o solucionar la actividad o tarea? desde esta interrogante se lograba concientizar hacia los procesos del estudiantado para la construcción del aprendizaje.

\section{RESULTADOS Y DISCUSIÓN}

Conforme con la experiencia y los resultados obtenidos en el estudio pedagógico, pueden considerarse los principios didácticos como prácticas inclusivas porque:

a. Originan una metodología de enseñanza activa. Para su desarrollo se implementan actividades innovadoras que llevaron al estudiante a vivir procesos cognitivos como: analizar, evaluar, sintetizar y comparar. Igualmente el estudiantado estaba en constante movimiento y comunicación con sus pares y docente.

b. El círculo como espacio de conversación diario, fomentó valores de respeto y tolerancia; fortaleciendo los lazos de amistad al crear sentimientos positivos de unos hacia otros porque lograban conocerse. A la docente le permitió crear vínculos fuertes con sus estudiantes generando un clima de confianza, ellos comentaban (en público o privado) situaciones personales, temas de interés, opiniones y sus necesidades. Esta práctica se aprovechó para incorporar en las actividades de clase temas de su preferencia o que apoyaran algunas vivencias personales relacionadas con sexualidad, resolución de conflictos, autoestima, valores, entre otros.

c. El aprendizaje cooperativo exteriorizó características de liderazgo, creatividad y autonomía en la mayoría de estudiantes. Los roles asignados llevaban a la persona a asumir un compromiso con el grupo y dar ideas creativas para enriquecer la tarea por cumplir. Muchos niños y niñas tomaban muy en serio la responsabilidad asignada así llegaban a ser "sujeto de control" para otros compañeros que se distraían o mostraban poca motivación por cumplir con su deber grupal. En ciertos momentos, utilizar la rúbrica en el trabajo grupal se convertía en un factor de inspección que le permitió a la docente garantizar el trabajo final de un grupo, porque al escribirse y calificarse la rúbrica en el cuaderno de cada asignatura los encargados podían supervisar el trabajo y el comportamiento de sus hijos e hijas en clase.

d. La enseñanza explícita, principalmente la agenda del día, se convirtió para la docente y estudiantes en un recurso importante para administrar el uso correcto de los tiempos de clase. Si las primeras lecciones las daba otra maestra, pedían a la profesora guía apenas ingresaba al aula colocar la agenda para poder predecir y organizar su día de estudio, incluso muchos dibujaban la agenda en sus cuadernos para llevar el control.

e. La activación de conocimientos previos se convirtió en una estrategia que estimulaba la motivación por la clase porque cada actividad otorgaba al estudiante detalles relevantes sobre el tema en 
desarrollo; ellos sentían que aportaban y que podían comentar sus experiencias personales. Este principio facultó al docente para advertir desde lo formativo el avance de cada estudiante, con sus comentarios se lograba percibir vocabulario de uso frecuente, quiénes investigaban sobre los temas de estudio, lecturas recreativas que realizaban, programas de televisión que veían, la música y los sitios web de su preferencia.

f. Concientizar los procesos de metacognición en cada estudiante, dio resultados positivos, para muchos de ellos era sencillo encontrar los procesos metacognitivos. Otros con apoyo de la docente o de un compañero, lograba advertir sus propias rutas cognitivas. Para los estudiantes que mostraban mayor dificultad se fortaleció la comprensión y uso de la metacognición desde la atención individual (docente-estudiante).

g. El modelaje cognitivo fue uno de los principios preferidos por el estudiantado porque manifestaron sentir que actuaban en un programa de televisión, entonces resaltaban características histriónicas y de humor en algunos de ellos. A los estudiantes les gustaba modelar desde el segundo nivel "participativo", algunos comentaban que era como asumir y dirigir la clase. Mediante el uso frecuente del modelaje cognitivo desde sus tres niveles, se lograron avances significativos en el uso de estrategias expuestas como la construcción de textos, la división y multiplicación abreviada, entre otros.

h. Entre los principales desafíos que enfrentó la docente al incorporar los principios didácticos en su metodología de enseñanza se encuentran:

- Los problemas propios de un trabajo por equipos. Un problema podían ser la baja disposición al trabajo de un integrante del equipo porque no deseaba relacionarse con alguno compañero en específico. Lo anterior requería vigilancia constante por parte de la docente hacia el cumplimiento de los roles asignados para evitar que el equipo se distrajera con frecuencia o discutieran entre sí. Otro problema podía ser que un equipo no lograba ponerse de acuerdo en la toma de decisiones, entonces la docente intervenía para ayudarles a organizarse o conversar en privado con algún miembro que no colaboraba. Por último la exposición era un momento no deseado para algunos estudiantes que temían hablar en público o que aún se les dificultaba ordenar sus ideas para explicarlas al exponer.

- La no comprensión de la metodología de trabajo por parte de los encargados de familia. Las clases eran muy dinámicas porque se aprovechaba el tiempo para la construcción del aprendizaje y no para copiar la materia de estudio, la docente antes de iniciar un tema entregaba resúmenes a los estudiantes y los cuadernos se utilizaban para escribir el trabajo grupal, las prácticas, los resúmenes elaborados por los niños y niñas, entre otros usos. Lo anterior preocupaba a algunos padres que manifestaban que al no ver los cuadernos llenos no comprendían que se hacía en la escuela todo el día. Un apoyo que permitió aminorar el conflicto fue realizar mensualmente reuniones de padres, donde se explicaba con detalles las actividades de clase, los tema por desarrollar y además se evacuaban consultas al respecto.

- Los cambios que conlleva la preadolescencia influía en muchas ocasiones en la motivación o disposición de los estudiantes para realizar algunas actividades de clase. Al inicio algunos no querían sentarse en el piso porque se ensuciaban el uniforme o sentían que era una actividad para niños pequeños. Se notaban cambios repentinos en el humor o tolerancia de algunos de ellos, entonces surgían discusiones y desacuerdos. Sus juegos en los recreos tendían a ser bruscos o formaban grupos cerrados de amigos que excluían a algún compañero o compañera. No obstante, para todas las situaciones siempre se buscaron solucionarse desde el diálogo directo con los involucrados, casi de forma inmediata la docente guía intervenía para prevenir que alguna pelea o discusión se saliera de control. 


\section{CONCLUSIONES}

La educación costarricense demanda procesos de enseñanza y aprendizaje que respondan a un currículo abierto que minimice las barreras de aprendizaje. Los principios didácticos constructivistas, como prácticas de aula, son inclusivos, activos, flexibles e innovadores; convirtiéndose en una opción viable para incorporar la educación inclusiva en la escuela actual. Desde esta experiencia, se concluye que implementar cambios en las metodologías de enseñanza permite subsanar factores de familia y del contexto educativo que influyen en la motivación y el aprendizaje de los estudiantes que cursan el cuarto grado. Estimular constantemente las habilidades sociales en el estudiantado reafirmó el uso de valores como la tolerancia, el respeto, el orden y la colaboración, así como el ejercicio de la ética; formando así, ciudadanos con un alto conocimiento de sus habilidades para la vida y para la convivencia en cualquier contexto social.

Los principios didácticos constructivistas pueden convertirse en una opción de cambio e innovación para los centros de enseñanza. Poseen una naturaleza sencilla ya que nace del sentir del docente, no requiere grandes costos o proyectos para aplicarlos, sólo demanda de una actitud de convencimiento por parte del docente, quién debe abandonar su papel de notable y asumir una nueva posición ante sus estudiantes, permitiéndole ser parte de un sistema de enseñanza apto para ellos, que considera sus diferencias y que los hace sentir incluidos y obtener éxito en su aprendizaje. Los principios didácticos permiten entender la diversidad como una oportunidad y no una desventaja para los procesos de enseñanza aprendizaje.

\section{REFERENCIAS}

Abarca, S. (2007). Frecuencia con que los (as) docentes de primaria del circuito 01 de la Dirección Regional de Educación de Cartago aplican los principios del constructivismo en el proceso de enseñanza-aprendizaje. Tesis de Maestría, Universidad Estatal a Distancia, San José, Costa Rica.

Arias, M., Coronado, V., Rolla, A., Romero, S., Rivadeneira, M. (2011). Didáctica de la lectoescritura 1. Para una construcción guiada de las competencias de lenguaje. San José, Costa Rica: EUNED.

Calero, M. (2008). Constructivismo pedagógico. Teorías y aplicaciones básicas. Distrito Federal, México: Alfaomega.

CAST (2008). Universal desing for learning guidelines version 1.0. Walkefield, MA: Autor.

Díaz, F., Hernández, R. (2002). Estrategias docentes para un aprendizaje significativo: una interpretación constructivista. Distrito Federal, México: McGraw Hill.

Escudero, J. y Martínez, B. (2011). Educación Inclusiva y cambio escolar. Revista lberoamericana de educación. Recuperado de http://webcache.googleusercontent.com/search?q=cache:XOW2K76uJlsJ:rieoei.org/rie55a03.pdf $+\& c d=2 \& h l=e s \& c t=c l n k \& c l i e n t=f i r e f o x-b$

Hernández, R. M. (2004). Mediación en el aula. Recursos, estrategias y técnicas didácticas. San José, Costa Rica: EUNED.

Jiménez, A. (2009). Psicología Constructivista. [Versión electrónica]. Enfoques Educativos 2009, 7 (37), 64-74.

López, G. (2008). Interacción y comunicación: hacia el constructivismo virtual Recuperado de http://e-constructivismo. blogspot.com/2008/10/interaccin-y-comunicacin-hacia-el.html

Ministerio de Educación Nacional. (2009). Estrategias apoyo a la gestión académica con enfoque inclusivo. Bogotá, Colombia. Autor. 
Muntaner, J. (2014). Prácticas inclusivas en el aula ordinaria. Revista de Educación Inclusiva. Recuperado de https://dialnet.unirioja.es/servlet/articulo?codigo=4772623

Programa Estado de la Nación. (2011). Tercer Informe Estado de la Educación. Capítulo 1: Sinopsis. Recuperado de http://www.estadonacion.or.cr/index.php/biblioteca-virtual/costa-rica/ educacion/sinopsis/informe-iii

Puchol, D. (2010). Elmodelado:definición, factores claves yámbito de aplicación en psicoterapia. Recuperado de http://www.psicologia-online.com/colaboradores/dpuchol/modelado.shtml

Rubio, A. \& Álvarez, A. (2010). Formación de Formadores después de Bolonia. Madrid, España: Díaz de Santos. 